Britta Renner/Ralf Schwarzer

\title{
Gesundheitspsychologie
}

\section{Geschichtliche Entwicklung}

Die Gesundheitspsychologie ist ein vergleichsweise neues, expandierendes Teilgebiet der Psychologie. Die Entstehung und Entwicklung dieser Disziplin sowie deren Forschungsaktivität kann am besten anhand der Gründung von Fachgesellschaften, Online-Diensten, wissenschaftlichen Zeitschriften sowie am Ausbildungsangebot verdeutlicht werden (vgl. Schwenkmezger/ Schmidt 1994).

Erstmalig als Fach etabliert wurde die Gesundheitspsychologie 1978, als innerhalb der American Psychological Association (APA) eine Division of Health Psychology gegründet wurde. An den Universitäten gehört das Fach Health Psychology inzwischen zu den Standarddisziplinen und ist innerhalb der Departments of Psychology angesiedelt, manchmal in Kombination mit anderen Teildisziplinen (z.B. "Health and Social Psycho$\log$ «). Rund acht Jahre später richtete die International Association for Applied Psychology (IAAP) ihre Fachgruppe Health Psychology ein. Ebenfalls 1986 wurde die European Health Psychology Society (EHPS) gegründet. Die Deutsche Gesellschaft für Psychologie (DGPs) hat 1992 die Fachgruppe Gesundheitspsychologie eingerichtet.

Diese Fachgruppe bietet offiziell bereits seit 1995 einen Online-Dienst im World Wide Web an (URL: http://userpage.fuberlin.de/ gesupsy). Dieser Online-Dienst wurde allein im Juni 1998 von rund 900 Internetsurfern in Anspruch genommen. Psychologische Institute wie beispielsweise in Berlin, Mainz und Landau bieten solche Online-Dienste zum Thema Gesundheitspsychologie an. Eine Übersicht liefert der Server "psychologie.de" (http://psychologie.de/katalog/rubriken/040200.html).

Ferner wurden insbesondere in den 90er Jahren verschiedene Zeitschriften und Buchreihen gegründet, deren Fokus auf gesundheitspsychologischen Themen liegt. So erscheint seit 1992 eine Buchreihe mit dem Titel "Gesundheitspsychologie" sowie seit 1993 die Zeitschrift für Gesundheitspsychologie. Maßgebliche Zeitschriften in englischer Sprache sind Health Psychology (1982ff); Psychology and Health (1987ff), Journal of Health Psychology (1996ff), Journal of Occupational Health Psycho- 
logy (1996ff) und das British Journal of Health Psychology (1996ff) sowie das Jahrbuch »International Review of Health Psychology« (1992ff).

Der Fortschritt und Entwicklungsstand der Gesundheitspsychologie läßt sich auch am Ausbildungsangebot ablesen (vgl. dazu http://userpage.fu-berlin.de/ gesupsy/ausbildung/index.html). Eine Befragung der Fachgruppenmitglieder im Oktober 1997 ergab, daß in Deutschland an 34 Universitäten das Fach Gesundheitspsychologie innerhalb des Diplomstudienganges Psychologie gelehrt wird (http://userpage.fu-berlin.de/ gesupsy/mitteilungen/972/befrag.html). Im Vergleich zum Jahre 1994 sind damit allein 23 neue Standorte hinzugekommen.

Gesundheitsbegriff und

Paradigmenwechsel
Diese wenigen ausgewählten Daten machen deutlich, daß sich das neue Fach Gesundheitspsychologie insbesondere seit den $80 \mathrm{er}$ Jahren mit großer Geschwindigkeit entwickelt hat. $\mathrm{Zu}$ dieser raschen Entwicklung haben verschiedene Faktoren beigetragen (vgl. Bishop 1994; Petrie/Weinman 1997; Schwenkmezger/ Schmidt 1994). Von Bedeutung war die Veränderung des Gesundheitsbegriffs. Früher wurde Gesundheit nur als die Abwesenheit von Krankheit definiert, heute hingegen in einem mehr positiven Sinne auch als psychisches und physisches Wohlbefinden. Ein weiterer wichtiger Aspekt ist der Paradigmawechsel. Das biomedizinische Modell wurde durch ein biopsychosoziales Modell abgelöst. Im Fokus steht damit nicht mehr nur der physische Aspekt von Gesundheit und Krankheit, sondern auch psychische und soziale Faktoren finden Berücksichtigung. Ferner hat eine Veränderung der primären Morbiditäts- und Mortalitätsursachen dazu beigetragen, daß das individuelle Gesundheits- und Krankheitsverhalten stärker in den Blickpunkt rückte. Die größte Herausforderung für die Medizin lag in der Vergangenheit in der Bekämpfung akuter Infektionskrankheiten, da diese damals die häufigste Todesursache in den Industrienationen darstellten. Durch die Entdeckung der Bedeutung der Hygiene und der Antibiotika gelang es jedoch, diese Krankheiten erfolgreich zurückzudrängen. Heute stellen chronische Erkrankungen des Herzkreislaufsystems sowie Krebserkrankungen die primären Todesursachen in den westlichen Ländern dar. Da äußeren Lebensumständen und dem Verhalten eine zentrale Rolle bei der Entstehung und Bewältigung dieser Krankheiten zukommt, kann die Gesundheitspsychologie einen wichtigen Beitrag zur Förderung und Erhaltung von Gesundheit leisten. Abschließend sei noch hinzugefügt, daß auch die Sichtweise des "Patienten" einem Wandel unterlag. Der Patient wird nicht 
mehr als passives Subjekt verstanden, das medizinischen Anweisungen folgt. Vielmehr suchen Menschen in zunehmendem Maße aktiv gesundheitsrelevante Informationen und erwarten, daß ihre Einschätzungen und Bedürfnisse berücksichtigt werden. Die Gesundheitspsychologie untersucht solche gesundheits- und krankheitsrelevanten Kognitionen sowie deren Einfluß auf Interaktionsprozesse zwischen Patient und Gesundheitssystem.

\section{Gegenstände der Gesundheitspsychologie}

Bishop (1994) schlägt eine sehr breite Definition von Gesundheitspsychologie vor. Danach handelt es sich um ein Teilgebiet der Psychologie, welches sich mit der dynamischen Beziehung zwischen Verhalten, psychischen Zuständen und der körperlichen Gesundheit beschäftigt. Deutlich wird anhand dieser Definition, daß der Fokus der Gesundheitspsychologie auf dem körperlichen Befinden liegt und dadurch ein weites Spektrum menschlichen Verhaltens und Erlebens angesprochen wird. Bishop (1994) thematisiert vier Perspektiven der Gesundheitspsychologie:

1. Förderung und Erhaltung von Gesundheit

Das Augenmerk liegt hier auf der Frage, wie das Gesundheits- bzw. Risikoverhalten von Menschen, die noch nicht erkrankt sind, beeinflußt werden kann.

2. Prävention und Behandlung von Krankheiten

Hier steht neben der Förderung von gesundem Verhalten die Frage im Vordergrund, welche Bedingungen eine konstruktive Bewältigung von gesundheitlichen Bedrohungen ermöglichen.

3. Identifikation von psychischen Faktoren, die zur Entstehung von Krankheiten beitragen

Erforscht wird auch die Rolle von psychischen und sozialen Faktoren für die Genese von Krankheiten. Zahlreiche Studien haben gezeigt, daß Streß und mangelnde soziale Unterstützung zu Erkrankungen führen können.

4. Einfluß der Interaktion zwischen Gesundheitssystem und Patient auf das individuelle Gesundheitsverhalten

Analysiert wird hier, wie beispielsweise die Bereitschaft, medizinischen Anweisungen zu folgen, oder die Genesungsrate davon beeinflußt wird, wie Arzt und Patient miteinander umgehen. 
Schwarzer (1997) spricht bei der Definition des Faches noch weitere Facetten an, indem er auch die Rehabilitation, die Bestimmung von Risikoverhaltensweisen und die Verbesserung des Systems gesundheitlicher Versorgung berücksichtigt. Abbildung 1 stellt diese Definition zusammenfassend dar, wobei Teilaspekte, die über die Begriffsbestimmung von Bishop (1994) hinausgehen, grau unterlegt sind.

Abb. 1: Definition des Gegenstands der Gesundheitspsychologie nach Schwarzer (1997)

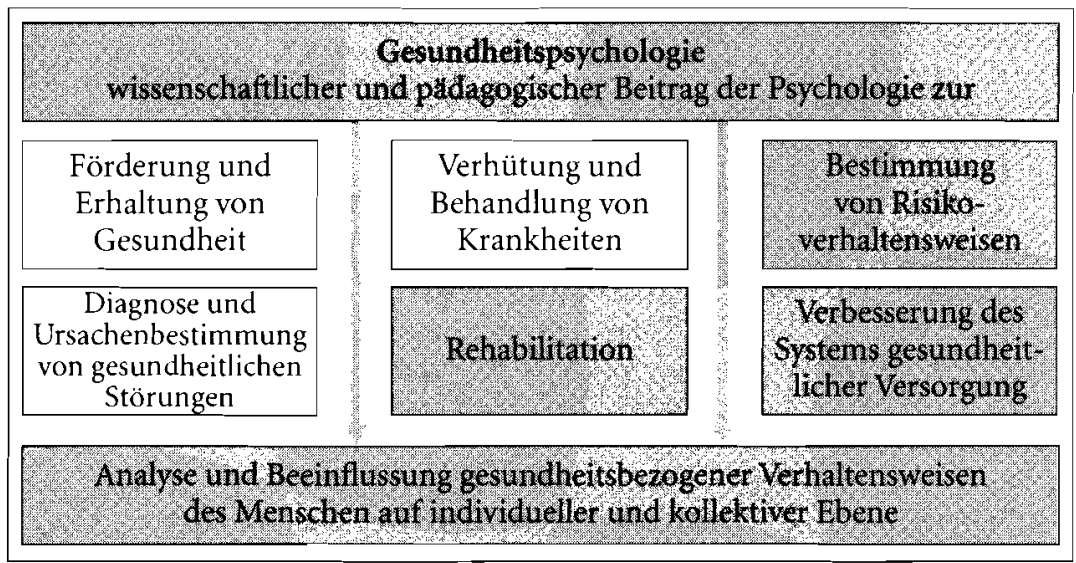

Zusammenfassend kann festgehalten werden, daß die Gesundheitspsychologie sich mit den Entstehungsbedingungen und der Prävention von gesundheitlichen Störungen und Risikofaktoren befaßt, sowie mit Determinanten des Krankheitsbewältigungs- und Genesungsprozesses. Dies geschieht unter Rückgriff auf andere psychologische Fächer und unter besonderer Berücksichtigung protektiver Faktoren von Gesundheit. Gesundheitspsychologie ist damit eine anwendungsbezogene, integrative Disziplin innerhalb der Psychologie im Unterschied zur Verhaltensmedizin, die sich als interdisziplinär versteht.

\section{Forschungsmethoden der Gesundheitspsychologie}

In der empirischen Forschung zu gesundheitspsychologischen Themen kommen vielfältige Methoden zur Anwendung. 
Die Befragung ist sicherlich die am häufigsten angewendete Methode, um gesundheitsbezogene Einstellungen und Verhaltensweisen zu erfassen. Grundsätzlich kann hier unterschieden werden zwischen der Interviewtechnik und der Fragebogentechnik, wobei der prägnanteste Unterschied zwischen beiden in der Erhebungssituation liegt. Schriftliche Befragungen werden im allgemeinen als anonymer erlebt, was die Tendenz verringert, sozial erwünscht zu antworten. Darüber hinaus sind sie in einem höheren Maße standardisiert, was sich günstig auf die Vergleichbarkeit der Daten auswirkt.

Systematische Beobachtungsverfahren werden seltener eingesetzt als Befragungen. Der Vorteil dieser Methode gegenüber der Befragungstechnik liegt darin, daß sie durch die Untersuchungsteilnehmer weniger verfälscht werden als verbale Selbstdarstellungen. Fisher, Fisher, Misovich, Kimble und Malloy (1996) fragten Studentinnen danach, wie schwierig es für sie sei, Safer Sex zu praktizieren. Um die Selbstangaben zu validieren, wurden die Studentinnen bei einer Diskussion über Safer Sex gefilmt. Diese Aufnahmen wurden anschließend von geschulten Ratern danach beurteilt, wie gut es der jeweiligen Untersuchungsteilnehmerin gelang, ihr Gegenüber davon zu überzeugen, künftig Safer Sex zu praktizieren. Zwischen den Selbstangaben und den Einschätzungen der Beobachter bestand eine signifikante Übereinstimmung.

Wenn man Menschen nach ihren Risikoverhaltensweisen fragt, ist ein häufiges Problem das sogenannte »under-reporting (Harris/Middleton 1994). Dies kann teilweise darauf zurückgeführt werden, daß insbesonders komplexe Verhaltensweisen, wie beispielsweise Ernährungsgewohnheiten, nicht genau erinnert und eingeschätzt werden können. Loftus, Feinberg und Tanur (1985) merken an, daß die Art und Weise, in der Informationen im Gedächtnis organisiert sind, die Erinnerungsgenauigkeit beeinflußt. Wenn nun Menschen angeben sollen, wie häufig sie in einem bestimmten Zeitraum einen Arzt aufgesucht haben oder bestimmte Nahrungsmittel verzehrten, dann können sie die tatsächliche Häufigkeit nicht genau erinnern. In diesem Fall werden kognitive Heuristiken (Faustregeln) genutzt, um diese zu schätzen. Heuristiken sind meist jedoch ungenau, so daß das Antwortverhalten verzerrt wird. Hoorens und Harris (1998) konnten einen Zeitrahmen-Effekt (time span effect) zeigen. Demnach werden generell weniger protektive sowie riskante Verhaltensweisen berichtet, wenn sich die Angaben auf einen langen (1 Jahr) im Vergleich zu einem kurzen Zeitraum (1 Monat) beziehen. Ein weiteres Problem stellt die Tendenz dar, sozial

Typische Verzerrungen 
Physiologische Messungen erwünscht zu antworten. Dies gilt insbesondere für "loaded questions «, d. h. Fragen, die eine emotionale Konnotation aufweisen. Denkt man beispielsweise an den Konsum von Alkohol, dann findet sich eine Diskrepanz zwischen der berichteten Menge an konsumiertem Alkohol und der im gleichen Zeitraum verkauften Menge. Gleiches gilt für den Selbstbericht an positiven Gesundheitsverhaltensweisen, allerdings findet sich hier naturgemäß eher eine Tendenz zum »over-reporting (Klein 1996). Das Ausmaß protektiven Verhaltens (z.B. Kondombenutzung) wird meist zu positiv dargestellt. Ferner kann eine Befragung bereits eine (unerwünschte) Intervention darstellen, die das interessierende Verhalten beeinträchtigt. So kann allein die Frage nach gesundheitsbezogenen Vorsätzen eine Intentionsbildung anregen. Fühlt sich eine Person hingegen unbeobachtet, so wird sie sich möglicherweise anders verhalten.

Physiologische Messungen werden eingesetzt, um Interdependenzen zwischen physiologischen und psychologischen Merkmalen aufzudecken. Ein Beispiel dafür ist die Wirkung von Streß auf physiologische Parameter (z.B. Herzfrequenz), die Erkrankungen hervorrufen oder den Krankheitsverlauf ungünstig beeinflussen können. Experimentelle Untersuchungen konnten nachweisen, daß psychischer Streß mit einer erhöhten Infektionsanfälligkeit der oberen Luftwege, Erkältungskrankheiten und mit einer Herpes-Virus-Infektion assoziiert war (Cohen/ Tyrrell/Smith 1991; Cohen/Williamson 1991; Glaser et al. 1987). Ferner kann Ärger die Entwicklung von kardiovaskulärer Hyperaktivität und Atherosklerose begünstigen (Schwenkmezger 1994; Weber 1994). Ein weiteres Beispiel ist die Erforschung des Zusammenhangs zwischen der Vergabe eines Krankheitsetiketts wie "Hypertonie" und der subjektiven Befindlichkeit. Problematisch hierbei ist, daß insbesondere Laborsituationen für die Untersuchungsteilnehmer eine ungewöhnliche Situation darstellen, auf die sie häufig mit erhöhter Erregung und Anspannung reagieren. Deutlich wird dies anhand des Phänomens des »weißen Kittels«. Allein die Konfrontation mit einem Arzt kann bei vielen Menschen bewirken, daß ihr Blutdruck ansteigt. Physiologische Messungen werden auch als Ergänzung zur Befragung genutzt. So können Selbstangaben beispielsweise hinsichtlich des Tabakkonsums durch die Messung der CotininKonzentration im Urin überprüft werden. Allein die Ankündigung, daß Aussagen über das eigene Rauchverhalten durch Meßgeräte verifiziert werden sollen, führt zu einer besseren Präzision dieser Selbstberichte. 
Experimentelle Methoden können danach unterschieden werden, ob die Untersuchungsteilnehmer den einzelnen Bedingungen nach Zufall zugewiesen werden (»echtes« Experiment) oder die Zuteilung anhand situativer Umstände determiniert wird (Quasi-Experiment). So wird die kognitive und emotionale Verarbeitung von Risikoinformationen sowohl experimentell als auch quasi-experimentell untersucht. Im ersten Fall wird z.B. der Blutdruck gemessen, anschließend erhalten die Untersuchungsteilnehmer eine falsche Rückmeldung über ihren gemessenen Blutdruck. Croyle (1990) teilte seine Untersuchungsteilnehmer nach Zufall entweder der Bedingung »erhöhter Blutdruck « oder der Bedingung "normaler Blutdruck« zu. Anschließend erfragte er die Einschätzungen der persönlichen Gesundheitsbedrohung aufgrund des mitgeteilten Blutdruckwertes. Leppin (1994) hingegen nutzte einen quasi-experimentellen Ansatz, indem sie ihren Untersuchungsteilnehmerinnen ihren tatsächlichen Blutdruck mitteilte. In beiden Untersuchungen zeigte sich, daß Personen, die erfuhren, ihr Blutdruck sei erhöht, im Vergleich zu jenen, deren Blutdruck als unbedenklich bezeichnet worden war, die gesundheitlichen Implikationen einer Hypertonie weniger schwerwiegend einschätzten. Anhand beider Methoden konnte damit nachgewiesen werden, daß Menschen Risikoinformationen in defensiver Weise verarbeiten. Der Vorteil des echten Experiments gegenüber dem quasi-experimentellen liegt darin, daß Störfaktoren wie das Vorwissen oder die Krankengeschichte kontrolliert werden und damit die kausale Interpretation der Ergebnisse erleichtert wird.

Ferner können auch natürliche Experimente der Beantwortung von gesundheitspsychologischen Fragestellungen dienen. In diesem Fall tritt eine Intervention ein, die nicht auf eine Manipulation des Forschers zurückgeht. Beispielsweise können das Bewältigungsverhalten nach Katastrophen und die gesundheitlichen Beeinträchtigungen der Opfer verglichen werden mit den Merkmalen solcher Menschen, die nicht diesem kritischen Lebensereignis ausgesetzt waren.

Der Vorteil von Experimenten liegt darin, daß Aussagen über die Kausalität von Variablenbeziehungen möglich sind. Korrelative Assoziationen reichen nicht aus, um eine Beeinflussung von Krankheit und Gesundheit durch psychologische Prozesse anzunehmen. Bei vielen Fragestellungen der Gesundheitspsychologie ist eine experimentelle Manipulation von Variablen und eine randomisierte Zuweisung von Personen zu experimentellen Gruppen indes nicht möglich oder ethisch nicht vertretbar. So können Implikationen einer Hospitalisation oder schwerwie- 
Kausalanalysen und Längsschnittstudien

genden Erkrankung aus praktischen und ethischen Gründen nicht im Rahmen eines Experiments untersucht werden. Dies macht nicht-experimentelle Analysen notwendig. Ferner sind Aussagen über gesundheitsbezogene Einstellungen und Verhaltensweisen einer ganzen Population nicht möglich. Hierfür sind Befragungen an Stichproben notwendig.

Auch nicht-experimentelle Kausalanalysen nehmen einen wichtigen Platz in der Methodologie dieses Arbeitsgebietes ein (vgl. dazu Hodapp 1994). Anhand von Querschnittdaten, die auf keiner experimentellen Manipulation beruhen, kann keine Aussage über die Richtung der Variablenbeziehung getroffen werden. Dies macht vielmehr Längsschnittstudien für die Gesundheitspsychologie unerläßlich, da sich nur auf diese Weise Prozesse in ihrer zeitlichen Dynamik und die zugrundeliegenden Faktoren darstellen lassen (Hodapp 1994). Überdies kann so die Stabilität von Merkmalen oder die Vorhersage eines Verhaltens aufgrund des früheren Verhaltens erfaßt werden. Allerdings können Konfundierungen mit Drittvariablen auch bei Längsschnittuntersuchungen dazu führen, daß Verknüpfungen über die Zeit bestehen, die nicht auf den Einfluß der zuerst gemessenen Variablen zurückgehen. Aus diesem Grund ist es notwenig, eine Kontrollgruppe zu berücksichtigen, die keine Intervention erhalten hat. Fisher et al. (1996) versuchten, gesundheitsbezogene Kognitionen und sexuelles Risikoverhalten anhand einer gezielten Intervention günstig zu beeinflussen. Um den Interventionseffekt statistisch nachweisen zu können, brachten sie ein längsschnittliches Kontrollgruppendesign zur Anwendung. Die Hälfte der nach Zufall ausgewählten Studentinnen nahm an einem insgesamt sechsstündigen Workshop teil, in dessen Rahmen individuelle Defizite hinsichtlich des Wissens über HIV und AIDS, der Motivation, Kondome zu benutzen, sowie Schwierigkeiten bei der praktischen Anwendung von Kondomen und Verhaltensdefizite thematisiert und abgebaut werden sollten. Vor dem Workshop sowie einen Monat danach wurde diese Gruppe ausführlich befragt. Eine Kontrollgruppe, die nicht an dem Workshop teilnahm, wurde ebenfalls im gleichen Zeitraum zweimal befragt. Die Analysen zeigen, daß die Interventionsgruppe im Vergleich zur Kontrollgruppe über die Zeit hinweg günstige Veränderungen in allen vier Bereichen erzielte. In diesem Fall kann von einem Einfluß der Intervention auf gesundheitsbezogene Kognitionen und Verhaltensweisen gesprochen werden. 


\section{Anwendungsbereiche der Gesundheitspsychologie}

Die primäre Prävention ist darauf ausgerichtet, bei gesunden Menschen entweder Risikoverhaltensweisen (z.B. Rauchen, Alkohol- und Fettkonsum) zu verringern und gesundes Verhalten (z.B. sportliche Aktivität) zu fördern, oder darauf, die Entwicklung von Risikoverhaltensweisen von vornherein zu verhindern. Ziel ist es, das Auftreten von Krankheiten zu verhüten. Die sekundäre Prävention hingegen soll die Progression einer bereits bestehenden Erkrankung verhindern, indem bereits möglichst früh erste Krankheitsanzeichen erkannt und behandelt werden. Die tertiäre Prävention umfaßt kurative und rehabilitierende Maßnahmen. Die Gesundheitspsychologie kann zu allen drei Präventionsbereichen wertvolle Beiträge leisten. Um eine Systematisierung der gesundheitspsychologischen Anwendungsbereiche zu erleichtern, ist es hilfreich, zwischen Gesundheitsverhalten, das in erster Linie dem Bereich der primären Prävention zugeordnet werden kann, und Krankheitsverhalten, das dem Bereich der sekundären und tertiären Prävention zuzuordnen ist, zu unterscheiden.

\subsection{Gesundheit und Verhalten}

Was veranlaßt Menschen dazu, Risikoverhaltensweisen aufrechtzuerhalten oder Gesundheitsverhaltensweisen aufzubauen? Die Bedingungen gesundheitlich bedeutsamen Verhaltens stellen einen der zentralen Gegenstände der Gesundheitspsychologie dar. Dazu gehören die Wahrnehmung einer Bedrohung, Erwartungen hinsichtlich der eigenen Bewältigungskompetenz und der Handlungskonsequenzen sowie die Bildung einer Verhaltensintention.

\subsubsection{Ein Prozeßmodell des Gesundheitsverhaltens}

Zur theoretischen Erklärung des Gesundheitsverhaltens ist es sinnvoll, zwischen der Motivations- und der Volitionsphase zu unterscheiden. In der Motivationsphase geht es darum, zwischen verschiedenen alternativen Handlungszielen zu wählen und eine Bereitschaft zur Handlungsänderung zu entwickeln. Eine gesündere Lebensführung kann auf ganz unterschiedliche Art und Weise verwirklicht werden. So kann die Person sich dafür entscheiden, sich gesünder zu ernähren, körperlich aktiver zu werden oder mit dem Rauchen aufzuhören. Diese Entscheidungssituation hat nicht selten den Charakter eines Dilemmas, denn riskantes Verhalten wie z.B. Rauchen hat neben den gesundheitsschädigenden Wirkungen auch positive Konsequenzen, die 
das Verhalten verstärken. Dieser Prozeß mündet in einer Zielintention (z.B. »Ich will mit dem Rauchen aufhören«), die diesen Entscheidungskonflikt vorerst beendet. In der anschließ3enden

Volition Volitionsphase geht es darum, diese Zielintention zu realisieren. Hier können wiederum verschiedene Barrieren und Widerstände auftreten, die eine erfolgreiche Umsetzung verhindern oder erschweren. Nachdem das Verhalten in die Tat umgesetzt wurde, stellt sich die Schwierigkeit der Aufrechterhaltung gegen Widerstände. Dies ist insbesondere bei Verhaltensweisen kritisch, die eine regelmäßige Ausführung erfordern, um wirksam zu sein.

Neben dieser zeitlichen Phaseneinteilung sind auch situative Faktoren wie soziale Unterstützung (Leppin/Schwarzer 1997) zu berücksichtigen. Das Prozeßmodell soll nun etwas näher betrachtet werden (vgl. Abb. 2).

Abb. 2: Sozial-kognitives Prozeł 3 modell des Gesundheitsverhaltens von Schwarzer (1996)

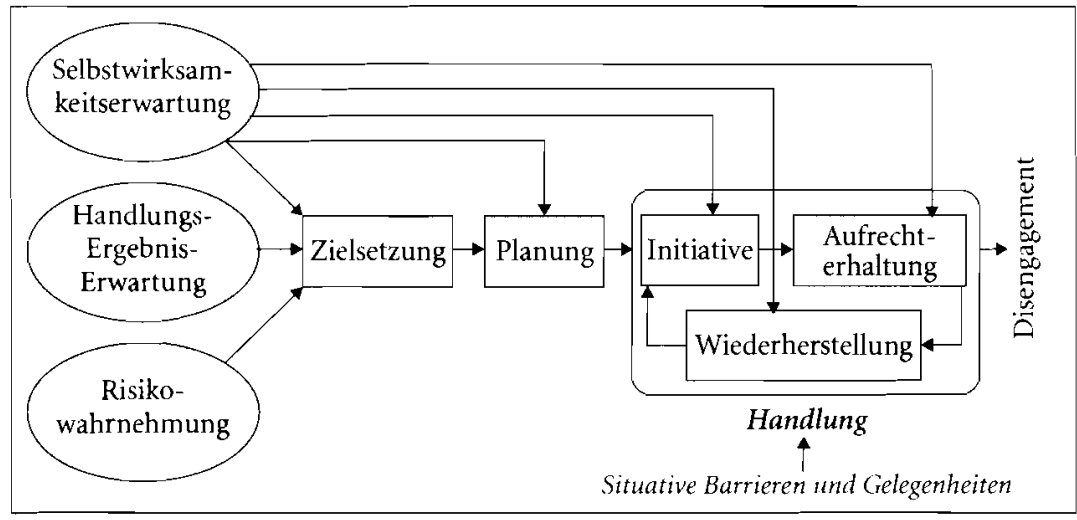

\subsubsection{Die Motivation zum Gesundheitsverhalten}

Risikowahr- Risikowahrnehmung: Die bewußte Wahrnehmung eines gesundnehmung heitlichen Risikos ist für den motivationalen Prozeß von Bedeutung. Diese Einschätzung der eigenen Verwundbarkeit (Vulnerabilität) wird meist anhand von Fragen operationalisiert wie "Für wie wahrscheinlich halten Sie es, daß Sie einmal an Lungenkrebs erkranken werden? «. Ferner ist das Ausmaß des wahrgenommenen Schweregrads der Erkankung ausschlaggebend dafür, wie hoch das eigene Risiko eingeschätzt wird. Es liegt auf 
der Hand, daß Erkrankungen, denen eine hohe Auftretenswahrscheinlichkeit, jedoch gleichzeitig nur ein geringer Schweregrad (z.B. Erkältung) zugeschrieben wird, nur zu einer geringen Risikowahrnehmung führen. Die Motivation zu einer Verhaltensänderung dürfte demnach nur gering ausgeprägt sein. Generell stellt sich in diesem Zusammenhang das Problem, daß das eigene Risiko häufig als zu gering oder zu optimistisch eingeschätzt wird (vgl. dazu Renner/Schwarzer, im Druck; Schwarzer/ Renner 1997). Weinstein (1980) bezeichnete die Tendenz, das eigene Risiko als unterdurchschnittlich zu bewerten, als optimistischen Fehlschluß. Selbst Menschen, die sehr gut über eine Gesundheitsgefahr informiert sind, unterliegen dem unrealistischen Optimismus. So erkennen Raucher in gleicher Weise wie Nichtraucher, daß Rauchen zu schweren Gesundheitsschäden führt. Dennoch halten sie das Lungenkrebsrisiko eines anderen Rauchers, der genauso viel und genauso lange raucht wie sie selbst, für höher als das eigene Risiko (Renner/Hahn 1996; Hahn/Renner 1998). Ein Risiko zu erkennen, heißt demnach nicht zwangsläufig, daß man sich selbst als gefährdet einschätzt. Zur Erklärung und Vorhersage von gesundheitsbezogenen Intentionen müssen deshalb noch andere kognitive Faktoren berücksichtigt werden.

Handlungsergebnis-Erwartungen: Allgemein sind Handlungsergebnis-Erwartungen (outcome expectancies) subjektive Vorstellungen über die Konsequenzen einer Handlung (Bandura 1997). Operationalisiert werden sie durch Aussagen der folgenden Art: »Wenn ich regelmäßig sportlich aktiv wäre, dann wäre ich weniger anfällig für Krankheiten«. Handlungsergebnis-Erwartungen (Konsequenzerwartungen) entsprechen damit Kontingenzwissen, das durch eine systematische Gesundheitsaufklärung vermittelt werden kann. Allerdings sind auch sie, wie die Wahrnehmung einer gesundheitlichen Gefährdung, nur notwendige, aber keine hinreichenden Vorausetzungen, um sich gesünder zu verhalten.

Selbstwirksamkeitserwartungen: Damit gesundheitsbezogene Vorsätze gebildet werden, muß die Person auch davon überzeugt sein, daß sie tatsächlich in der Lage ist, diese schwierigen Gesundheitshandlungen auch erfolgreich ausführen zu können. Die Überzeugungen von persönlichen Handlungskompetenzen werden auch als Selbstwirksamkeitserwartungen (perceived self-efficacy) bezeichnet (Bandura 1997). Darin kommt die Überzeugung zum Ausdruck, auch angesichts von Barrieren gesundheitsbezogene Handlungen ausführen zu können. Ein Beispiel für eine Operationalisierung wäre »Ich bin sicher, daß ich

Konsequenzerwartungen

Selbstwirksamkeit 
funktionaler Optimismus

Risikokommunikation mich auch dann fettarm ernähren kann, wenn meine Familie ihre fettreiche Ernährung beibehält «.

Eine optimistische Einschätzung der eigenen Handlungskompetenzen angesichts von Barrieren wird auch als funktionaler Optimismus bezeichnet (Schwarzer 1994; Schwarzer/Renner 1997), der die Intentionsbildung und Handlungsausführung begünstigt. Die Analyse der wahrgenommenen Selbstwirksamkeit gibt Hinweise für die Gestaltung von Gesundheitsförderungsmaßnahmen.

Die Motivationsphase umfaßt demnach drei Komponenten, die gemeinsam maßgebend für die Bildung einer Zielintention sind: die Risikowahrnehmung, die Handlungsergebnis-Erwartung und die Selbstwirksamkeitserwartung. Hier stellt sich die Frage, wie diese drei Einflußgrößen ineinander wirken. Die Risikowahrnehmung löst zu Beginn eine Suche nach Informationen und die Bewertung von Handlungen und deren Ergebnisse aus. Diese Ergebniserwartungen können einer Verhaltensänderung abträglich (»Wenn ich mich fettarm ernähre, schmeckt mir das Essen nicht mehr so gut «) oder förderlich sein (»Wenn ich mich fettarm ernähre, dann werde ich mein Herz-Kreislauf-Risiko verringern «). Letztlich müssen Risikowahrnehmungen und Ergebniserwartungen von einer ausreichenden Selbstwirksamkeitserwartung unterstützt werden, damit auch wirklich eine Zielintention ausgeprägt wird. Anhand einer längsschnittlichen Feldstudie (Berlin Risk Appraisal and Health Motivation Study, BRAHMS) wurde die Wirkung einer Risikokommunikation auf Gesundheitsverhalten untersucht (Renner/Hahn 1998). Personen mit einem Risiko (erhöhter Cholesterinwert) waren stärker motiviert, eine gesündere Lebensweise anzustreben und setzten diese häufiger in die Tat um als Personen ohne Risiko (normaler Cholesterinwert) oder Personen aus der Kontrollgruppe. Neben diesem Effekt der Risikokommunikation kam jedoch auch der Selbstwirksamkeitserwartung Bedeutung zu. Die Risikomitteilung wurde insbesondere dann konstruktiv wirksam, wenn die eigene Handlungskompetenz hoch eingeschätzt wurde. Schwarzer und Fuchs (1996) haben anhand von Längsschnittuntersuchungen zeigen können, daß die Intention, sich künftig gesünder zu ernähren, in erster Linie von den HandlungsergebnisErwartungen determiniert wird. Die Selbstwirksamkeitserwartung war der zweitstärkste und die Risikowahrnehmung der drittstärkste Prädiktor. Diese Untersuchungen bieten allerdings nur vorläufige Hinweise auf die kausale Ordnung der drei Merkmale im Motivationsprozeß. 


\subsubsection{Von der Intention zur Handlung}

"Der Geist ist willig, aber das Fleisch ist schwach « charakterisiert sehr gut die Lage von Personen, die die Motivationsphase erfolgreich mit einer Verhaltensintention abgeschlossen haben, dann aber auf der Stelle treten und die Umsetzung in aktives Gesundheitshandeln zögerlich vor sich herschieben. Diese zweite Phase wird als Volitionsphase bezeichnet. Sie läßt sich wiederum unterteilen in eine präaktionale und eine aktionale Phase. In der präaktionalen Phase wird die beabsichtigte Handlung im Detail geplant. Das Wie und Wo der Handlung wird festgelegt, wobei eine ganze Reihe alternativer Ausführungsideen generiert werden kann. Dann wird die Handlung initiiert (bzw. desaktiviert, wenn es um die Unterlassung eines kritischen Verhaltens geht). Mit Handlung ist hier sowohl ein Gesundheitsverhalten gemeint (z.B. Zähneputzen) als auch die Unterlassung eines Risikoverhaltens (z.B. Rauchen). Auf etwas Reizvolles oder Gewohntes zu verzichten, erfordert einen aktiven Bewältigungsprozeß mit der nötigen Volitionsstärke. Während der aktionalen Phase findet eine ständige Handlungsausführungskontrolle statt, in der es darum geht, sowohl die Handlung als auch die Intentionen gegenüber Distraktoren abzuschirmen. Metakognitive Abschirmund Durchhaltetendenzen können dafür sorgen, daß man nicht "abdriftet", nicht die Handlung unterbricht und nicht seine Aufmerksamkeit ständig anderen Dingen widmet. Gäbe es keine metakognitive Kontrollinstanz, würden wir den »roten Faden verlieren, uns von den augenblicklichen Umständen hin- und herschieben lassen und die Handlungsziele aus den Augen verlieren. Zu solchen Metakognitionen gehören auch »Durchhalteparolen « wie z.B. "Wenn ich jetzt aufgebe, war alles umsonst, was ich bisher schon an Anstrengung in dieses Gesundheitsverhalten investiert habe».

Um den Einfluß von Selbstwirksamkeitserwartung auf das Gesundheitsverhalten in verschiedenen Phasen zu beleuchten, haben Marlatt, Baer und Quigley (1995) die Unterscheidung von "action self-efficacy «, "coping self-efficacy « und "recovery selfefficacy« vorgenommen. In ihren Arbeiten zur Abhängigkeit von Nikotin, Alkohol und anderen Drogen haben sie herausgefunden, daß unterschiedliche kognitive Prozesse erforderlich sind, um Abstinenzziele hartnäckig und erfolgreich zu verfolgen. Beim ersten Versuch, das Risikoverhalten zu unterlassen, wird man von Optimismus beflügelt, indem man sich zutraut, aus eigener Kraft eine Pause von der Abhängigkeit einzulegen. Dann treten Schwierigkeiten auf, Hindernisse und Barrieren le-

Einfluß der Selbstwirksamkeit 
gen sich in den Weg. Hier geht es um Coping, also strategisches Bewältigungsverhalten, welches wiederum begünstigt wird, wenn man dafür entsprechende Selbstwirksamkeitserwartungen ausgebildet hat. Schließlich muß auch mit Rückschlägen oder Mißerfolgen gerechnet werden. Dafür müssen geeignete Wiederherstellungsstrategien erworben worden sein, also z.B. optimistische Interpretationsweisen, die das kritische Ereignis in einem nicht zu ungünstigen Licht erscheinen lassen.

\subsection{Krankheit und Verhalten}

Bisher wurde die Frage beleuchtet, welche psychischen Faktoren protektives Verhalten begünstigen. Im folgenden Abschnitt soll nun der Frage nachgegangen werden, wie sich Menschen angesichts einer Erkrankung verhalten. Der objektive Krankheitsstatus schlägt sich nicht notwendigerweise im subjektiven Befinden nie-

Illness und der. Im englischen Sprachgebrauch wird deshalb auch zwischen

Disease »illness « und »disease " unterschieden. »Illness « bezeichnet das subjektive Empfinden, krank zu sein, während »disease « eine medizinische Diagnose bezeichnet. Häufig besteht eine Diskrepanz zwischen der subjektiven und objektiven Einschätzung des Gesundheitszustandes, die unter anderem zu einer Art "Symptomparadox « führt. Auf der einen Seite nehmen Menschen trotz ernsthafter Beschwerden keine medizinische Hilfe in Anspruch, andererseits erfolgt rund die Hälfte der Hausarztbesuche aufgrund von Symptomen, die auf keine klinisch relevante Erkrankung zurückzuführen sind (Jennings 1986; Verbrugge 1985). Das Krankheitsempfinden und -verhalten wird offensichtlich nicht nur vom biologischen Zustand beeinflußt, sondern auch von kulturellen und sozialen Faktoren, situativen Umständen, Streß, Persönlichkeit und subjektiven Krankheitskonzepten.

Wahrnehmung von Symptomen
Der erste Schritt im Krankheitsprozeß ist meist die Wahrnehmung und Interpretation von Symptomen. Wie nehmen Menschen Symptome wahr, und was folgt daraus für das Krankheitsverhalten?

Wenn wir uns in einer negativen Stimmung befinden, scheinen wir nicht nur unseren Gesundheitszustand ungünstiger zu bewerten, sondern wir nehmen auch mehr Symptome wahr (Croyle/Uretsky 1987). Eine negative Stimmung steht in einem hohen positiven Zusammenhang mit der Symptomwahrnehmung, aber nur gering mit physiologischen Maßen (Watson/ Pennebaker 1991).

Ferner scheinen Menschen in einer reizarmen Umgebung häufiger Symptome wahrzunehmen. Pennebaker (1980) konnte 
nachweisen, daß bei langweiligen im Gegensatz zu interessanten Filmpassagen die Zuschauer häufiger husten. Menschen, die in erster Linie einer monotonen Erwerbstätigkeit nachgehen oder alleine leben, nehmen häufiger Aspirin und Schlaftabletten ein als Menschen, deren berufliche Tätigkeit interessant ist oder die mit anderen Menschen zusammenleben (Coburn 1975) - und dies, obwohl sie keine ungünstigeren physiologischen Meßwerte (z.B. Blutdruck, Herzrate) aufweisen. Wenn nur wenig externale Information vorliegt, dann wird offenbar die Aufmerksamkeit stärker internalen Prozessen zugewendet, und es werden häufiger Symptome wahrgenommen.

Normalerweise haben Menschen, auch wenn sie nicht erkrankt sind, zahlreiche physiologische Empfindungen - eine Art "Grundrauschen«. Welchen Symptomen Aufmerksamkeit geschenkt wird, hängt von ihren Erwartungen ab. So kann eine Krankheitsdiagnose die Suche nach konformen Symptomen stimulieren. Allein die Mitteilung, daß der Blutdruck erhöht sei, führt zu einer Veränderung der Symptomwahrnehmung (Baumann/Cameron/Zimmermann/Leventhal 1989). Studenten, denen ein erhöhter Blutdruck zurückgemeldet wurde, gaben mehr hypertone Symptome an und schätzten ihren Gesundheitszustand schlechter ein als diejenigen, die glaubten, ihr Blutdruck sei normal. Ein erhöhter Blutdruck wird meist mit unspezifischen Symptomen assoziiert, wie beispielsweise Kopfschmerzen, Schwindelgefühl oder Wärme (Pennebaker/Watson 1988). Diese Symptome treten häufig auf und werden im allgemeinen nicht als Anzeichen einer Krankheit interpretiert. Durch die Diagnose »Hypertonie « wird jedoch ein subjektives Krankheitsschema aktiviert, welches Vorstellungen über Symptome enthält, die mit einem erhöhten Blutdruck einhergehen. Dadurch wird die Aufmerksamkeit selektiv auf Symptome gelenkt, die mit dem Etikett Hypertonie übereinstimmen. Die Etikettierung (labeling) einer Person als krank kann die Symptomwahrnehmung somit in systematischer Weise beinflussen.

Die Verabreichung von Medikamenten oder Placebos kann ebenfalls zu einer selektiven Aufmerksamkeit gegenüber körperlichen Empfindungen führen. Menschen, die erkrankt sind, scheinen nach der Einnahme eines Medikaments ihre Aufmerksamkeit weniger den Krankheitssymptomen und mehr potentiellen Anzeichen der Genesung zu schenken (Ross/Olsen 1981).

Daß Erwartungen hinsichtlich des Auftretens von Symptomen die psychischen Reaktionen beeinflussen, demonstrierten auch Nerenz, Leventhal, Love und Ringler (1984). Sie interviewten Krebspatienten, die eine Chemotherapie erhielten. Patien-

Krankheitsschemata

Labeling 
Struktur von

Krankheitsschemata

ten, die eine unerwartet schnelle Verbesserung aufwiesen, litten erheblich mehr unter Distress als Patienten, deren Genesung graduell und erwartungsgemäß verlief. Die emotionalen Reaktionen auf die therapeutische Intervention wurden offenbar bestimmt durch das individuelle Krankheitsschema und der Ubereinstimmung des Genesungsverlaufs mit diesem. Krankheitsschemata stellen Wissensstrukturen dar, die Symptome und andere Krankheitsattribute umfassen. Wenn eine Person Symptome verspürt, wird sie versuchen, für diese ein passendes Krankheitsschema zu finden. Menschen nehmen damit nicht nur einzelne Symptome wahr, sondern stellen systematisch einen Zusammenhang zwischen diesen her. Spürt eine Person Fieber, Halsschmerzen und Kopfschmerzen, wird sie diese Beschwerden als Indikatoren für eine Erkältung werten. Krankheitsschemata enthalten allerdings nicht nur Information über Symptome, sondern auch über die Ursachen und die Dauer der Erkrankung sowie über kurative Maßnahmen (vgl. Leventhal et al. 1997). Diese vier Komponenten stellen offenbar eine relativ universelle interne Struktur von Krankheitsschemata dar. Die Ausprägung dieser Komponenten ist jedoch interindividuell sehr verschieden. Beispielsweise gibt es ganz unterschiedliche Vorstellungen über die Symptome und die Ursachen eines akuten Herzinfarkts.

Aufsuchen von medizinischer Hilfe

Krankheitsschemata beeinflussen das Verhalten. Problematisch ist dies, wenn sie falsche Annahmen enthalten und so dazu führen, daß nicht rechtzeitig medizinische Hilfe gesucht wird. Petrie und Weinman (1997) stellten fest, daß Personen mit einem akuten Herzinfarkt oft mehrere Stunden verstreichen lassen, bevor sie Hilfe suchen. Bedenkt man, daß 70 Prozent der Todesfälle innerhalb von vier Stunden auftreten, dann wird deutlich, warum erste Symptome von den Betroffenen richtig interpretiert werden müssen.

Der EntscheidungsprozeB, der zum Aufsuchen medizinischer Hilfe führt, kann nach Safer, Tharps, Jackson und Leventhal (1979) in drei Phasen unterteilt werden (vgl. Abb. 3). Die Einschätzungsphase umfaßt die Wahrnehmung von Symptomen und die Frage, ob diese tatsächlich eine Krankheit anzeigen. Wenn eine Person zu der Entscheidung gelangt, daß sie erkrankt ist, schließt sich die Krankheitsphase an. In dieser geht es um die Frage, ob medizinische Hilfe notwendig ist. Wenn auch diese positiv beantwortet wird, dann muB eine Person den Vorsatz bilden, Kontakt aufzunehmen und diesen verwirklichen (Nutzungsphase). In allen drei Phasen kann es zu Verzögerungen kommen, die dazu führen, daß wertvolle Zeit verstreicht. 
Abb. 3: Drei Stufen bis zur Kontaktaufnahme mit dem Gesundheitssystem (nach Safer, Tharps, Jackson \& Leventhal, 1979)

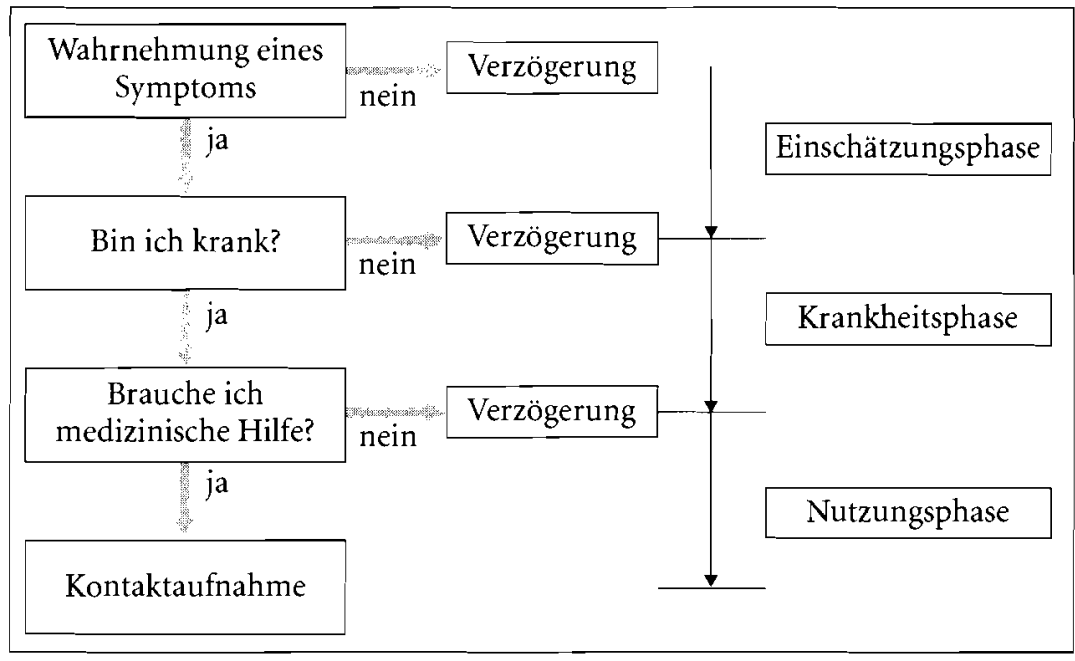

Safer et al. (1979) und Dracup et al. (1995) beobachteten bei Patienten, die kurz zuvor einen Myokardinfarkt erlitten hatten, daß die Einschätzungsphase deutlich länger als die Krankheitsphase dauerte. Die Einschätzungsphase war um so länger, je weniger salient und schmerzhaft die ersten Symptome waren. Ferner wurden die Symptome von einigen vermeintlich als Erschöpfungssymptome interpretiert, so daß sich die Betroffenen zunächst nicht krank fühlten. Dies war insbesondere der Fall, wenn eine hohe Arbeitsbelastung vorlag. Die Symptome entsprachen offenbar nicht eindeutig dem Krankheitsschema "Herzinfarkt", sondern paßten auch zum Schema "Erschöpfung «. Die situativen Umstände als Ursache für die körperliche Beeinträchtigung waren deshalb von entscheidender Bedeutung für die Interpretation der Symptome. Dies macht deutlich, daß eine Aufklärung, die lediglich über Symptome informiert, nicht ausreicht (Petrie/Weinman 1997). Vielmehr muß das subjektive Krankheitsschema sowie mögliche Überschneidungen mit anderen Schemata ebenfalls thematisiert werden.

Krankheitsschemata sind nicht nur entscheidend für das Aufsuchen medizinischer Hilfe, sondern auch dafür, ob eine BeAdherence handlung befolgt (Adherence) oder abgebrochen wird. Hypertoniker, die sich in ärztlicher Behandlung befinden, sind im allgemeinen gut über die Gefahren einer mangelnden Adhe- 
rence informiert. Dennoch nimmt nur rund ein Drittel ihre Medikamente entsprechend den ärztlichen Anweisungen ein. Meyer, Leventhal und Gutmann (1985) stellten fest, daß der wahrgenommene Einfluß der Medikamente auf die Symptome die Adherence moderierte. Hypertoniker, die der Überzeugung waren, daß die Medikamente ihre Symptome günstig beeinflussen, zeigten in 70 Prozent der Fälle eine gute Adherence. Von den Hypertonikern, die hingegen glaubten, daß die Medikamente ihre Symptome nicht beinflussen, nahmen nur 31 Prozent ihre Medikamente wie verordnet ein. Hier muß hinzugefügt werden, daß im allgemeinen eine Hypertonie symptomfrei ist. Eine Einnahme der Medikamente in Abhängigkeit von Symptomen ist deshalb unzuverlässig und generell nicht empfehlenswert. Der Mehrzahl der Befragten war dies auch bewußt, dennoch waren 88 Prozent der Meinung, daß sie selbst es sehr wohl spüren können, wenn ihr Blutdruck erhöht ist.

Ferner ist die Annahme über die Dauer der Hypertonie entscheidend dafür, ob es zu einem Abbruch der Behandlung kommt. Betrachtet man die Literatur zur Symptomwahrnehmung bei Hypertonikern, so zeigt sich, daß Hypertoniker häufig zu Beginn der Behandlung ihre Hypertonie als ein akutes und nicht als ein chronisches Leiden verstehen. Ein akutes Krankheitskonzept zeichnet sich im Gegensatz zu einem chronischen dadurch aus, daß die Erkrankung als zeitlich begrenzt und heilbar bewertet wird. Meyer et al. (1985) berichten ferner, daß Hypertoniker mit einem akuten Krankheitskonzept, bei denen die vermeintlichen Hochdrucksymptome nach einer gewissen Behandlungszeit verschwanden, dies meist als Heilung verstanden und die Medikamenteneinnahme abbrachen.

\section{Zusammenfassung und Perspektiven}

Die Gesundheitspsychologie ist eine junge Teildisziplin innerhalb der Psychologie, die stärker von der Grundlagenforschung als von der klinischen Anwendung bestimmt wird. Aufgrund ihres hohen Niveaus in Theoriebildung und Forschungsmethodik kann sie wichtige Impulse für die Gesundheitswissenschaften liefern. Sie befaßt sich mit dem menschlichen Erleben und Verhalten angesichts gesundheitlicher Risiken und Beeinträchtigungen, aber auch mit der Optimierung von Gesundheit im Sinne von Fitneß und Wellness. Im vorliegenden Beitrag wurden grob zwei Gegenstandsbereiche unterschieden: Gesundheitsverhalten und Krankheitsverhalten. Innerhalb dieser bei- 
den Bereiche gibt es wiederum viele Fragestellungen, die wegen ihres Umfanges hier nicht auch noch berücksichtigt werden konnten. Auf einige bisher noch nicht ausgeführte Aspekte sei daher an dieser Stelle hingewiesen. Ihnen kommt für die $\mathrm{Zu}$ kunft besondere Bedeutung zu.

Persönlichkeitsbedingte Erkrankungen: Schon in der älteren Psychosomatik ist man der Annahme nachgegangen, daß bestimmte Krankheiten überzufällig häufig mit einem bestimmten Persönlichkeitsprofil einhergehen. Die neuere gesundheitspsychologische Forschung hat dazu eine Reihe wichtiger Befunde zutage gefördert (Friedman 1998; Traue 1998). So war man seit den 60er Jahren bei der Ursachenbestimmung des Herzinfarkts dem sogenannten Typ A-Verhalten auf der Spur. Damit bezeichnet man ein Verhaltensmuster, das durch ehrgeiziges Leistungsstreben, Konkurrenzorientierung, Ungeduld, Zeitdruck, Feindseligkeit, Ärger, Aggressivität und explosive Sprechweise gekennzeichnet ist. Tatsächlich hat man in aufwendigen prospektiven Längsschnittstudien Zusammenhänge mit der Herzinfarkt-Inzidenzrate gefunden. Diese konnten jedoch später nicht mehr so deutlich repliziert werden. Nach heutigem Kenntnisstand ist anzunehmen, daß einzelne Komponenten dieses Verhaltensmusters die Entstehung des Herzinfarktes begünstigen, insbesondere Feindseligkeit, Ärgerausdruck und zynisches Mißtrauen.

Auch für die Krebserkrankung hat man vorauslaufende Merkmale der Persönlichkeit identifizieren können. Am ehesten scheinen depressive oder anti-emotionale Menschen, die sich von anderen abhängig machen und zugleich konfliktscheu sind, dafür prädestiniert zu sein. Man muß jedoch bei solchen Aussagen immer bedenken, daß mehrere Einflußgrößen für den viele Jahre dauernden pathogenen Prozeß von Bedeutung sind und daß es hier um die statistische Erklärung von Unterschiedlichkeit (Varianz) im Auftreten des Krankheitsmerkmals geht, nicht aber um Rückschlüsse auf den Einzelfall.

Strefs und Streßbewältigung: In der Gesundheitspsychologie wird Streß als ein potentiell krankmachender Prozeß angesehen. Streß meint dabei weder den Reiz noch die Reaktion, sondern vielmehr einen interaktiven Vorgang, bei dem eine Person angesichts einer kritischen Situation Einschätzungsprozesse (Kognitionen) vornimmt (Schwarzer 1993). Dabei werden Situationsgefahren zu den Bewältigungsressourcen in Beziehung gesetzt. Aus Kognitionen erwachsen dann emotionale und physiologische Reaktionen sowie Bewältigungsanstrengungen (Coping). Diese Sichtweise ist sehr verschieden von den Streßtheorien, die

Persönlichkeit und Krankheit
Streßbewältigung 
Personale und

soziale

Ressourcen

in Medizin und Naturwissenschaft vorherrschen. Ob jemand aufgrund von Streß krank wird oder nicht, hängt nicht nur von der streßreichen Situation ab, sondern auch von den Ressourcen, Einschätzungen und den zum Einsatz gebrachten $\mathrm{Co}^{-}$ pingstrategien sowie deren Erfolg. Langanhaltende, schwere Streßepisoden, wie zum Beispiel bei Krankheit und Tod eines geliebten Partners, bergen somit ein hohes Potential für einen pathogenen Prozeß, der sich in Morbidität und Mortalität niederschlagen kann. Nach einer Verwitwung z.B. ist die Lebenserwartung des verbleibenden Partners - statistisch gesehen - reduziert.

Einer der Mechanismen, die dem zugrundeliegen, ist die Immunsuppression. Wenn der Organismus durch Krisen und Depressionen belastet ist, wird das Immunsystem geschwächt, so daß sich Infektionskrankheiten und Tumorneubildungen häufiger beobachten lassen. Dies ist Gegenstand der Psychoneuroimmunologie (Hennig 1998).

Ressourcen: Um die Geschicke des Lebens bewältigen zu können, braucht man Ressourcen, darunter die persönlichen und sozialen Ressourcen. Zu ersteren zählt man objektive Kompetenzen und subjektive Kompetenzerwartungen (Selbstwirksamkeits-Erwartungen). Damit ist die Überzeugung gemeint, streßreiche Anforderungen aufgrund eigener Fähigkeit und Anstrengung bewältigen zu können. Welche Rolle dies für das Gesundheitsverhalten spielt, wurde weiter oben beschrieben. Bei der Krankheitsbewältigung hat sich dieses Merkmal ebenfalls als einflußreich erwiesen. Die Rehabilitation nach Herzinfarkt oder Bypassoperation verläuft günstiger bei Patienten mit hoher Selbstwirksamkeits-Erwartung als bei solchen mit niedriger Selbstwirksamkeits-Erwartung (vgl. Schröder 1997).

Von den sozialen Ressourcen weiß man seit langer Zeit, daß sie den Genesungsverlauf günstig beeinflussen können. Patienten, die gut in ihr soziales Netzwerk eingebettet sind und die sich unterstützt fühlen, werden im Durchschnitt schneller gesund.

Intervention Gesundheitsförderungs-Programme: Bei der Gestaltung und Bewertung von Interventionsprogrammen sind die Erkenntnisse der Gesundheitspsychologie von großem Nutzen. Will man zeitgemäß, effektiv und ökonomisch vorgehen, so muß man zunächst feststellen, in welcher psychischen Phase der Änderungsmotivation sich die Adressaten für ein solches Programm befinden. Wer sich zum Beispiel noch nie darüber $\mathrm{Ge}-$ danken gemacht hat, das Rauchen aufzugeben, muß ganz anders angesprochen werden als jemand, der es schon mehrfach vergeblich versucht hat. Gleichzeitig müssen die verfügbaren 
personalen und sozialen Ressourcen ins Kalkül gezogen werden. Es gibt moderne Konzepte davon, wie ein solches maßgeschneidertes Programm aussehen kann (Dijkstra/De Vries/Roijackers/ van Breukelen 1998). Der Mangel der meisten Förderungsansätze liegt darin, daß keine umfassende Prozeßtheorie zugrundeliegt und keine methodisch anspruchsvolle Evaluation stattfindet, in die auch die Analyse von differentiellen Effekten und von Nebenwirkungen einfließt. Für die Zukunft bedarf es hier innovativer Maßnahmen, denn der Primärprävention von Erkrankungen kommt die höchste Bedeutung zu. Dies gilt nicht nur mit dem Ziel der Vermeidung von individuellem Leid, sondern auch im Hinblick auf die volkswirtschaftliche Seite des Problems. Die Gesellschaft kann sich auf Dauer keine technisch perfekte und personell aufwendige kurative Medizin für alle Bürger leisten. Nur durch Vorbeugung läßt sich dem Problem einer immer älter werdenden Population, die von Multimorbidität gekennzeichnet ist, entgegentreten. Die Gesundheitspsychologie verfügt über tragfähige Konzepte, mit denen man primärpräventive Gesundheitsprogramme entwickeln kann.

\section{Literatur}

Bandura, A.: Self-efficacy. The exercise of control, New York 1979 - Baumann, L. J./Cameron, L. D./ Zimmerman, R. S./Leventhal, H.: Illness representations and matching symptoms, Health Psychology, 8, 1989, 449-469-Bishop, G. D.: Health Psychology. Integrating mind and body, Boston $1994-$ Coburn, D.: Job-worker incongruence. Consequences for health, Journal of Health and Social Behavior, 16, 1975 , 198-212-Cohen, S. / Tyrrell, D. A. / Smith, A. P.: Psychological stress and susceptibility to the common cold, New England Journal of Medicine, 325, 1991, 606-612 - Cohen, S./Williamson, G. M.: Stress and infectious disease in humans, Psychological Bulletin, 109, 1991, 5-24-Croyle, R. T.: Biased appraisal of high blood pressure, Preventive Medicine, 19, 1990, 40-44-Croyle, R. T./Uretsky, M. B.: Effects of mood on self-appraisal of health status, Health Psychology, 6, 1987, 239-253 - Dijkstra, A./De Vries, H./ Roijackers, J./van Breukelen, G.: Tailored interventions to communicate stage-matched information to smokers in different motivational stages, Journal of Consulting and Clinical Psychology, 66 (3), 1998, 4258 - Dracup, K./Moser, D. K./Eisenberg, M./Meischke, H./Alonzo, A. A./Braslow, A.: Causes of delay in seeking treatment for heart attack symptoms, Social Science and Medicine, 40, 1995, 379-392 - Fisher, J. D./Fisher, W. A./Misovich, S. J./Kimble, D. L./Malloy, T. E.: Changing AIDS risk behavior. Effects of an intervention emphasizing AIDS risk reduction information, motivation, and behavioral skills in a college student population, Health Psychology, 15, 1996, 114-123 - Friedman, H. S.: Self-healing personalities, in: H. S. Friedman (Ed.): Encyclopedia of mental health (pp. 453-459), San Diego (CA) 1998 - Glaser, R./Rice, J./Sheridan, J./Fertel, R./Stout, J./Speicher, C./Pinsky, D./Kotur, M./Post, A./Beck, M./KiecoltGlaser, J.: Stress-related immune suppression. Health implications. Brain, Behavior, and Immunity, 1 , 1987, 7-20 - Hahn, A./Renner, B.: Perceptions of health risks. How smoker status affects defensive optimism, Anxiety, Stress, and Coping, 11, 1998, 93-112 - Harris. P./Middleton, W.: The illusion of control and optimism about health: On being less at risk but no more in control than others, British Journal of Social Psychology, 33, 1994, 369-378-Hennig, J.: Psychoneuroimmunologie, Göttingen 1998 - Hodapp, V.: Kausalmodelle bei nicht-experimentellen Daten, in: P. Schwenkmezger/L. R. Schmidt (Hrsg.): Lehrbuch der Gesundheitspsychologie, Stuttgart 1994, S. 119-132 - Hoorens, V./Harris, P.: Distortions in reports of health behaviors. The time span effect and illusory superiority, Psychology and Health, 13, 1998,451-466 - Jennings, D.: The confusion between disease and illness in clinical medicine, 
Canadian Medical Association Journal, 135, 1986, 865-870 - Klein, W. M.: Maintaining self-serving social comparisons. Attenuating the perceived significance of risk-increasing behaviors, Journal of Social and Clinical Psychology, 15,1996, 120-142 - Leppin, A.: Bedingungen des Gesundheitsverhaltens, Weinheim 1994 - Leppin, A./Sctwarzer, R.: Sozialer Rückhalt, Krankheit und Gesundheitsverhalten, in: R. Schwarzer (Hrsg.): Gesundheitspsychologie. Ein Lehrbuch, Göttingen 1997, 2. Aufl., S. 349-373 Leventhal, H./Benyanini, Y./Brownlee, S./Diefenbach, M./Leventhal, E. A./Patrick-Miller, L./Robtitailee, C.: Illness representations. Theoretical foundations, in: K. J. Petrie/J. A. Weinman (Eds.): Perception of health and illness, Amsterdam 1997, pp. 19-45 - Loftus, E. F./Feinberg, S. E./Tanur, J. M.: Cognitive psychology meets the national survey, American Psychologist, 40, 1985, 175-180-Marlatt, G. A./Baer, J. S./Quigley, L. A.: Self-efficacy and addictive behavior, in A. Bandura (Ed.): Self-efficacy in changing societies, New York 1995, pp. 289-315-Meyer, D./Leventhal, H./Gutmann, M.: Common-sense models of illness: The example of hypertension, Health Psychology, 4, 1985, 115-135-Nerenz, D. R./Leventhal, H./Love, R. R./Ringler, K. E.: Psychological aspects of cancer chemotherapy, International Review of Applied Psychology, 33, 1984, 521-529-Pennebaker, J. W.: Perceptual and environmental determinants of coughing, Basic and Applied Social Psychology, 1, 1980, 83-91 - Pemebaker, J. W./Watson, D.: Blood pressure estimation and beliefs among normotensives and hypertensives, Health Psychology, 7, 1988, 309-328-Petrie, K. J./Weinman, J. A.: Illness representations and recovery from myocardial infarction, in: K. J. Petrie/J. A. Weinman (Eds.): Perception of health and illness, Amsterdam 1997, pp. 44l $-461-$ Renmer, B./Hahn, A.: Stereotype Vorstellungen über eine gefährdete Person und unrealistisch optimistische Risikoeinschätzungen, Zeitschrift für Gesundheitspsychologie, 4, 1996, 220-240 - Renner, B./Hahn, A.: Screenings als Maßnahme der Gesundheitsförderung - eine Längsschnittstudie, Psychomed, 10, 1998, 47-54-Renner, B.,Schwarzer, R.: Gesundheit. Selbstschädigendes Handeln trotz Wissen, in: H. Mandl/J. Gerstenmaier (Hrsg.): Die Kluft zwischen Wissen und Handeln. Empirische und theoretische Lösungsansätze, Göttingen (im Druck) - Ross, M./Olsen, J. M.: An expectancy-attribution model of the effects of placebos, Psychology Review, 88, 1981, 408-437-Safer, M. A./Tharps, Q.JJackson, T./Leventhal, $H$. : Determinants of three stages of delay in seeking care at a medical clinic, Medical Care, 17, 1979, 11-29-Schröder, K.: Self-regulation competence in coping with chronic disease, Berlin 1997 Schwarzer, R.: Streß, Angst und Handlungsregulation, Stuttgart 1993 - Schwarzer, R.: Optimism, vulnerability, and self-beliefs as health-related cognitions: A systematic overview, Psychology and Health, 9, 1994, 161-180 - Schwarzer, R.: Psychologie des Gesundheitsverhaltens, Göttingen 1996 - Schwarzer, R.: Gesundheitspsychologie, Göttingen 1997 - Schwarzer, R./Fuchs, R.: Self-efficacy and health behaviours, in: $M$. Conner/P. Norman (Eds.): Predicting health behaviour, Buckingham (UK) 1996, pp. 163-196 - Schwarzer, R./Renner, B.: Risikoeinschätzung und Optimismus, in: R. Schwarzer (Hrsg.): Gesundheitspsychologie, Göttingen 1997, S.43-66 - Schwenkmezger, P.: Gesundheitspsychologie. Die persönlichkeitspsychologische Perspektive, in: P. Schwenkmezger/L. R. Schmidt (Hrsg.): Lehrbuch der Gesundheitspsychologie, Stuttgart 1994, S. 46-64 - Schwenkmezger, P./Schmidt, L. R.: Gesundheitspsychologie. Alter Wein in neuen Schläuchen?, in: P. Schwenkmezger/L. R. Schmidt (Hrsg.): Lehrbuch der Gesundheitspsychologie, Stuttgart 1994 - Traue, H. C.: Emotion und Gesundheit, Heidelberg 1998 Verbrugge, L. M.: Triggers of symptoms and health care, Social Science and Medicine, 20, 1985, 855-876 - Watson, D./Pennebaker, J. W. Situational, dispositional, and genetic bases of symptom reporting, in: $J$. A. Skelton/R. T. Croyle (Eds.): Mental representations in health and illness, New York 1991, pp. 60-84Weber, H.: Ärger. Psychologie einer alltäglichen Emotion, Weinheim 1994 - Weinstein, N. D.: Unrealistic optimism about future life events, Journal of Personality and Social Psychology, 39, 1980, 806-820 